\title{
Daten und Informationen
}

\section{Die Situation}

„Die EDV muss unsere Informationen klassifizieren!" Solchen Forderungen sehen sich IT-Leiter regelmäßig ausgesetzt. Häufig ist aber die EDV-Abteilung mit einer solchen Anforderung schlichtweg überfordert - nicht aus Mangel an Kompetenz, sondern weil die Verarbeitung von Informationen, im Gegensatz zu Daten, nicht in den Zuständigkeitsbereich der EDV-Abteilung gehört.

Im allgemeinen Sprachgebrauch wird fast nie eine exakte Unterscheidung zwischen den Begriffen Informationen und Daten vorgenommen - im Gegenteil: So werden beispielsweise die Abteilungsbezeichnungen „IT“ und „EDV“ meist synonym verwendet.

Ein sauberes Verständnis und eine klare Vereinbarung für den Gebrauch der Begriffe „Daten“ und „Informationen“ sind jedoch wichtige Grundlagen für den Erfolg vieler Projekte in den Bereichen Informations- und IT-Sicherheit sowie Datenschutz.

\section{Die Informationen}

Für den Begriff „Information“ gibt es keine einheitliche, abgestimmte wissenschaftliche Definition. Nach gängigen Definitionen des Begriffs in der Informatik ist Information von puren Daten durch ihren Sinngehalt zu unterscheiden. Streng genommen kann Information daher nur durch menschliche Interpretation von $\mathrm{Da}$ ten entstehen und das, was technisch gesehen auf Computern aller Couleur abläuft, ist Datenverarbeitung - nicht etwa Informationsverarbeitung.

Wird Daten also in einem bestimmten Kontext eine inhaltliche Bedeutung zugemessen, werden sie zu Informationen. Daher ist auch der Begriff des Schutzes „personenbezogener Daten“ irreführend, da sich ein personenbezogenes Merkmal erst ergibt, wenn Daten interpretiert, mithin also zu Informationen werden.

Mit dem Blick auf die IT-Sicherheit sind besonders die Informationen von Interesse, denen eine Bedeutung für Geschäftsprozesse zukommt, beispielsweise die spe- zifischen Umsätze eines Unternehmens in einem Geschäftsbereich, aber auch Kundendaten (korrekt eigentlich: Kundeninformationen, ein Begriff, der im Deutschen mit einer ganz anderen Bedeutung belegt ist).

\section{Die Daten}

Der Mensch kann zahllose Informationen in seinem Gehirn verarbeiten, ohne ihnen eine greifbare, nach außen vermittelbare Gestalt geben zu müssen. Sollen die Informationen allerdings zwischen Menschen ausgetauscht oder der automatisierten Verarbeitung mittels Maschinen zugänglich gemacht werden, bedarf es der Darstellung der Informationen in einer für alle Beteiligten zugänglichen bzw. maschinenlesbaren Form. Genau deshalb werden Informationen durch Daten dargestellt, die sich dann beispielsweise in Dateien, Datenbanken oder auf Datenträgern speichern sowie über Netze übermitteln lassen.

Sind sich alle Beteiligten über die Interpretation dieser Daten und ihren Sinngehalt einig, können - vermittelt über einen Datenaustausch - auch Informationen übermittelt, bearbeitet oder gespeichert werden.

Bei der maschinellen Verarbeitung ist jedoch immer zu bedenken, dass Daten außerhalb des zugehörigen Kontextes ihre Bedeutung verlieren. Beispielsweise lässt sich ohne Kontextinformation nicht entscheiden, ob die Zeichenkette „AFFE“ ein Säugetier oder die Zahl 45054 repräsentiert. ${ }^{1}$

\section{Die Mitspieler}

Damit Geschäftsprozesse reibungslos funktionieren benötigen alle beteiligten Fachbereiche ihre spezifischen Informationen, die sie in der Regel aus Daten ableiten. Die Verantwortung für deren Quali-

1 Eben dies ist auch der Kern des „Präsentationsproblems" bei digitalen Signaturen, die (anders als ein Mensch) Daten und nicht Informationen unterschreiben; siehe Kunz/Pordesch/Schmidt, DuD 12/2003 und Fox, DuD 7/1998. tät und den Informationsfluss tragen die Personen, die für die verbundenen Prozesse verantwortlich sind.

Da der Umgang mit elektronischen Daten häufig ein großes Spezialwissen erfordert, werden diese meist ausgesuchten Spezialisten treuhändisch übergeben. Diese Spezialisten, im Allgemeinen als EDVoder IT-Abteilung bezeichnet, arbeiten jedoch gerade nicht mit Informationen, sondern ausschließlich mit den Daten, die ihnen von den Prozessverantwortlichen übergeben werden. Für die Erfüllung ihrer originären Aufgaben hat und benötigt die EDV-Abteilung in der Regel keine konkrete Vorstellung von der Bedeutung der Daten für die Geschäftsprozesse. Denn ihr fehlt das erforderliche Zusatzwissen, um den Informationsgehalt der Daten ermessen zu können. Außerdem ist der Zugriff auf insbesondere sensible Daten durch das EDV-Personal (Administratoren) in der Regel sogar unerwünscht, sofern er nicht der Aufrechterhaltung technischer Abläufe dient.

\section{Die Erkenntnis}

Da es für die Fachbereiche jedoch wichtig ist, dass ihre Informationen angemessen geschützt werden, müssen sie die Schutzbedürftigkeit selbst festlegen und dokumentieren. Denn nur sie wissen um deren Bedeutung.

Erst auf der Grundlage einer solchen Festlegung durch die Fachabteilung kann die EDV-Abteilung angemessene Schutzmaßnahmen für die von ihr treuhändisch verwalteten Daten ableiten und ergreifen.

Die Herausforderung der beschriebenen Arbeitsteilung besteht also in der $\mathrm{Zu}$ sammenarbeit zwischen Fachbereichen und EDV-Abteilung, von der Ermittlung der Schutzbedürftigkeit von Informationen repräsentierenden Daten bis hin zur Festlegung und Umsetzung geeigneter Schutzmaßnahmen. Diese Zusammenarbeit kann in der Praxis durch eine moderierte Vorgehensweise sinnvoll unterstützt werden. Für diese Moderationsrolle bietet sich ein Informationssicherheitsmanager an. 